\title{
A Water Flow Control with OnLine Neurofuzzy Controller using a Dynamic Learning Rate
}

\author{
Hugo Gomes $^{1}$, Carolina Ferreira $^{1}$, Everthon Oliveira ${ }^{1}$, Agnaldo Reis ${ }^{2}$, Walmir Caminhas ${ }^{1}$ \\ ${ }^{1}$ Programa de Pós-Graduação em Engenharia Elétrica - UFMG \\ Av. Antônio Carlos 6627, 31270-901, Belo Horizonte, MG, Brasil \\ ${ }^{2}$ Departamento de Engenharia de Controle e Automação \\ Universidade Federal de Ouro Preto (UFOP) - Ouro Preto, MG - Brazil \\ \{hugonog, carolinazmf, everthonsol, caminhas\}@ufmg.br, reis@ufop.edu.br
}

\begin{abstract}
This paper describes an ONFC (OnLine Neurofuzzy Controller) application with a dynamic learning rate to control the water flow of a real plant. A revision of ONFC is presented and the $O N F C_{D w}$ version is used, which has an action that minimizes the increase in the difference between the controller weights. The dynamic learning rate used to update the controller weights is described and the results of experiments performed in a water flow control process are presented, comparing the results with the PID controller used in the process.
\end{abstract}

Resumo. Este artigo apresenta a utilização do ONFC (OnLine Neurofuzzy Controller) com taxa de aprendizagem dinâmica para controle da vazão de água de uma planta real. É apresentada uma revisão do controlador ONFC e empregada a versão ${ }_{\text {ONFC }}$ Dw a qual possui integrada uma ação que minimiza o aumento da diferença entre os pesos do controlador. É descrita a abordagem utilizada na atualização dos pesos do controlador com o uso de uma taxa de aprendizagem dinâmica. Por fim são apresentados resultados de experimentos realizados para controlar a vazão de água que alimenta um tanque, comparando o desempenho com o controlador PID empregado no processo.

\section{Introdução}

Os controladores com ação Proporcional, Integral e Derivativa (PID) são ainda os mais usados nos processos industriais, devido a sua rápida e fácil implementação e poucos parâmetros de ajuste. Existem vários métodos para ajuste dos parâmetros desses controladores, sendo que todos eles geram parâmetros eficientes para uma faixa restrita de operação do processo e para as condições operacionais do momento em que o método de sintonia foi aplicado [Ogata 2003].

Todavia, em última análise, a grande maioria dos processos industriais são não lineares e/ou com parâmetros variantes no tempo. Por esses motivos, para manter as especificações de desempenho, frequentemente os controladores PID apresentam baixo desempenho e exigem novas sintonias de seus parâmetros, seja por mudanças nas características do processo ou do ponto de operação do controlador.

Com o propósito de automatizar o ajuste dos parâmetros, controladores utilizando inteligência computacional vêm sendo empregados, devido ao fato de apresentarem capacidade de adaptação às mudanças do processo e facilidade de ajuste dos parâmetros. 
Dentre os controladores baseados em inteligência computacional estão os que utilizam lógica nebulosa, redes neurais ou neurofuzzy. Este último agrega as boas características das redes neurais e dos sistemas nebulosos e estão entre as estruturas de controle inteligente mais utilizadas [Precup and Hellendoorn 2011], como Adaptative Network based Fuzzy Inference Systems (ANFIS) [Jang et al. 1997] e Neo-Fuzzy-Neuron (NFN) [Yamakawa et al. 1992].

Um exemplo bem sucedido de controle baseado em NFN foi proposto por [Gouvêa 2005]. O chamado Online Neurofuzzy Controller (ONFC) consiste em um controlador de estrutura simples, que ajusta o peso das funções de pertinência de forma online, baseado no erro.

O ONFC é um controlador nebuloso adaptativo, cuja dinâmica se assemelha a um controlador PI adaptativo como demonstrado em [Carvalho 2010]. Apresenta baixo custo computacional, simplicidade de ajuste de seus parâmetros e capacidade de operação em processos com características não lineares. Este controlador já foi empregado com sucesso em controle de velocida de de motores [Gouvêa 2005], controle de processos não lineares [Pires 2007], em controle de processos petroquímicos [Carvalho 2010], controle de posição de robôs [dos Santos et al. 2016] e controle de temperatura de fornos [Ferreira 2017].

Um importante parâmetro de projeto do controlador é a taxa de aprendizado, que ajusta a velocidade de adaptação e o desempenho do controle em malha fechada. $\mathrm{Na}$ literatura de controle adaptativo são encontrados estudos que mostram a influência deste parâmetro na estabilidade do processo controlado. No caso do ONFC são aplicadas taxas de adaptação constantes ajustadas de maneira empírica.

Apresenta-se no presente artigo uma aplicação do controlador ONFC em um processo real para regulação de vazão de água em uma tubulação por meio de uma bomba. É apresentada ainda uma nova proposta de ajuste dinâmico da taxa de aprendizagem a fim de melhorar o desempenho do ONFC.

Este artigo está organizado da seguinte forma. Na Secção 1 foi apresentada uma introdução do tema em estudo. O controlador ONFC é tratado na Secção 2. A taxa de aprendizado dinâmica na Secção 3. A metodologia empregada e os resultados obtidos são discutidos nas Secções 4 e 5, respectivamente. Por fim, as conclusões do trabalho são apresentadas na Seção 6.

\section{Revisitando o ONFC}

O potencial de manuseio de incertezas e de controle de sistemas complexos proporcionado pela lógica nebulosa combinados com as características de aprendizagem e adaptação das Redes Neurais Artificiais (RNA) geram as classes de controladores neurofuzzy.

O Online Neurofuzzy Controller (ONFC) utiliza a estrutura da rede Neo Fuzzy Neuron (NFN) [Yamakawa et al. 1992] que no caso de sistemas com uma entrada e uma saída é equivalente ao mecanismo de inferência Takagi Sugeno de ordem zero, com duas regras fuzzy do tipo:

- Se erro (e) é $A_{1}$ então a ação $u$ é $u_{1}=w_{1}$;

- Se erro (e) é $A_{2}$ então a ação $u$ é $u_{2}=w_{2}$, 
sendo $A_{1}$ e $A_{2}$ dois conjuntos nebulosos representados pelas funções de pertinência triangulares e complementares $\mu_{1}(e)$ e $\mu_{2}(e)$, na qual $\mu_{1}+\mu_{2}=1$. A estrutura de controle é apresentada na Figura 1, enquanto na Figura 2 são apresentadas as funções de pertinência triangulares complementares.

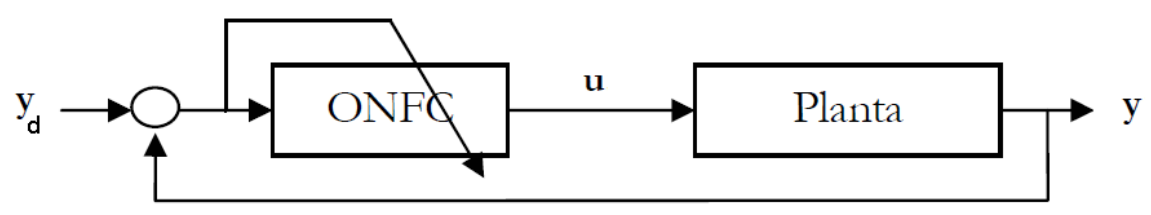

Figure 1. Estrutura de controle com o ONFC - [Pires 2007].

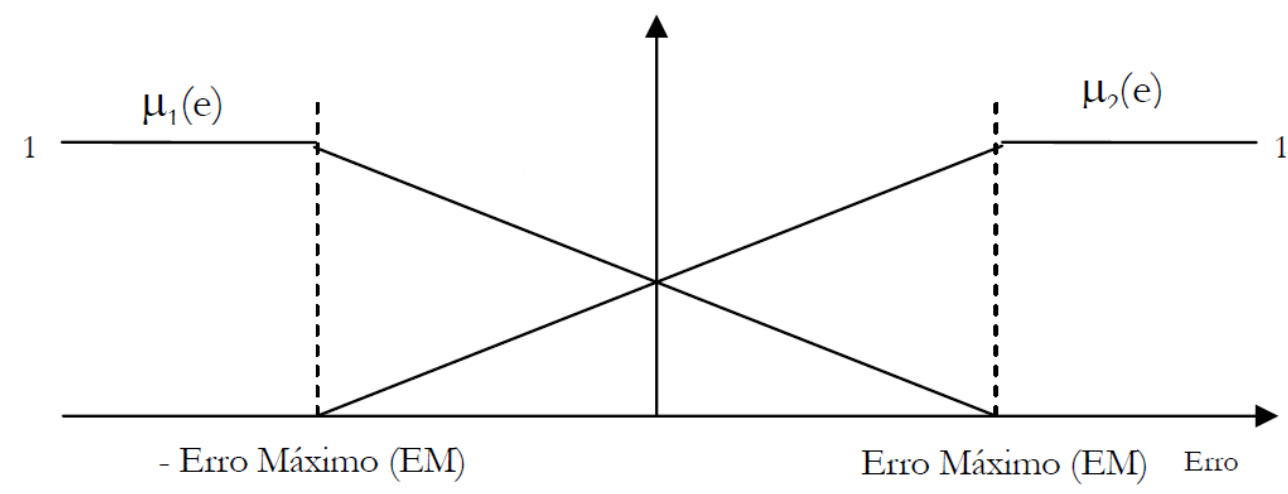

Figure 2. Funções de pertinência utilizadas no ONFC - [Pires 2007].

O erro, conforme definido na Equação 1, é a diferença entre o setpoint $\left(y_{d}\right)$ e a variável controlada $(y)$. O cálculo da ação de controle resultante $(u)$ é dado pela equação 2 e os cálculos para as funções de pertinência pelas equações 3 e 4 , onde $E M$ o erro máximo definido no universo de discurso.

$$
\begin{aligned}
& e=y_{d}-y \\
& u=\frac{\mu_{1}(e) u_{1}+\mu_{2}(e) u_{2}}{\mu_{1}(e)+\mu_{2}(e)} \therefore u=\mu_{1}(e) w_{1}+\mu_{2}(e) w_{2}
\end{aligned}
$$

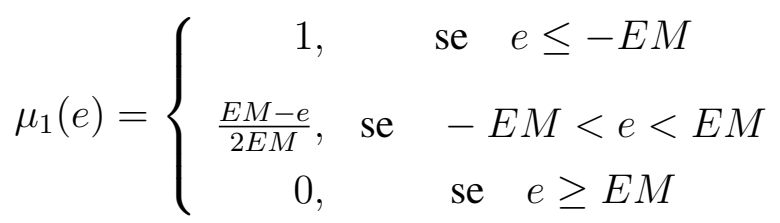

$$
\begin{aligned}
& \mu_{2}(e)=\left\{\begin{array}{cc}
0, & \text { se } e \leq-E M \\
\frac{E M+e}{2 E M}, & \text { se } \quad-E M<e<E M \\
1, & \text { se } e \geq E M
\end{array}\right.
\end{aligned}
$$


O processo de aprendizagem do ONFC ocorre pelo método do gradiente descendente (Equação 5), ou seja, a atualização dos pesos $w_{1}$ e $w_{2}$ da rede NFN que o constitui é feita a partir da minimização de uma função custo $J$ definida como o erro quadrático (Equação 6) a cada instante de amostragem k do sistema de controle:

$$
\begin{gathered}
w_{i}^{k}=w_{i}^{k-1}-\alpha_{0} \frac{\partial J^{k}}{\partial w_{i}^{k}} \\
\min J=\frac{1}{2} e^{2}
\end{gathered}
$$

A obtenção da regra de ajuste dos pesos é feita a partir da derivada da função custo em relação aos pesos (regra da cadeia), como é apresentado nas Equações 7 a 11.

$$
\begin{gathered}
\frac{\partial J}{\partial w_{i}}=\frac{\partial J}{\partial e} \frac{\partial e}{\partial y} \frac{\partial y}{\partial u} \frac{\partial u}{\partial w_{i}} \\
\frac{\partial J}{\partial e}=2 \frac{1}{2}(e)=e \\
\frac{\partial e}{\partial y}=-1 \\
\frac{\partial u}{\partial w i}=\mu_{i} \\
w_{i}^{k}=w_{i}^{k-1}+\alpha_{0} \frac{\partial y}{\partial u} \mu_{i}^{k} e^{k}
\end{gathered}
$$

A derivada parcial $\frac{\partial y}{\partial u}$ que representa a variação da saída da planta em relação à variação da entrada normalmente não é conhecida a priori. Conforme foi proposto por [Gouvêa 2005], apenas o conhecimento do sentido do ganho da planta (sendo 1 se a ação é direta e -1 se a ação é reversa) é suficiente para agrupar a taxa de aprendizagem $\alpha_{0} \mathrm{e}$ $\frac{\partial y}{\partial u}$ em uma única constante $\alpha$, obtendo-se a equação de ajuste final:

$$
w_{i}^{k}=w_{i}^{k-1}+\alpha \mu_{i}^{k} e^{k}
$$

Considerando que o erro não ultrapassa os limites do universo de discurso, definidos quando ocorre a seleção do parâmetro $E M$, são realizadas substituições na equação da ação do controlador [Pires 2007], permitindo escrever:

$$
u_{k}=\frac{w_{2}^{k}-w_{1}^{k}}{2 E M} e^{k}+\frac{w_{2}^{k}+w_{1}^{k}}{2}
$$

A inicialização dos pesos $w_{10}$ e $w_{20}$ é feita com valores nulos e a taxa de aprendizagem $\alpha$ em cada interação é fixa. Após manipulação algébrica em [Pires 2007], tem-se: 


$$
u_{k}=\frac{\alpha \cdot \sum_{i=1}^{k} e_{i}^{2}}{2 E M^{2}} \cdot e_{k}+\frac{\alpha}{2} \cdot \sum_{i=1}^{k} e_{i}
$$

Analisando a Equação 14, é possível fazer uma comparação do ONFC com um controlador PI adaptativo. Observa-se que há duas parcelas, uma relacionada ao erro no instante $k$ e outra parcela relacionada ao somatório do erro. Assim, podem ser visualizadas equivalências com as constantes proporcional $K_{P}$ e integral $K_{I}$ :

$$
\begin{gathered}
K_{P}=\frac{\alpha \cdot \sum_{i=1}^{k} e_{i}^{2}}{2 E M^{2}} \\
K_{I}=\frac{\alpha}{2}
\end{gathered}
$$

A Equação 15 apresenta uma constante proporcional adaptativa, que começa com valor zero e vai aumentando de acordo com o erro quadrático. A ação proporcional é ponderada pelo parâmetro $\alpha$ e também inversamente proporcional ao parâmetro $E M$. A Equação 16 apresenta uma constante integral fixa, não adaptativa, que depende única e exclusivamente do parâmetro $\alpha$. Percebe-se então que o parâmetro $\alpha$ influencia diretamente no desempenho do controlador e na estabilidade do mesmo.

Outra observação importante decorrente dessa análise é que a constante proporcional $K_{P}$ vai aumentando com o somatório do erro quadrático. Em processos reais, com a presença de ruídos, essa constante aumentaria indefinidamente podendo chegar a valores muito elevados. No ONFC esse problema faz com que os consequentes cresçam em direções opostas, aumentando a diferença entre eles. Tal situação tem particular importância uma vez que pesos muito altos aumentam a possibilidade de instabilidade no controle do processo e diminuem a contribuição da parcela integral do controlador. $\mathrm{Na}$ formulação do ONFC original, algumas estratégias foram criadas para contornar esse problema [Gouvêa 2005]. Uma solução foi a formulação do $\mathrm{ONFC}_{D w}$ [Carvalho 2010] que é o Online Neurofuzzy Controller modificado, com a inclusão da ponderação da diferença dos pesos das funções de pertinência $(D w)$.

$\mathrm{O}_{\mathrm{ONFC}} \mathrm{Dw}$ é uma evolução do ONFC que tende a limitar os pesos da rede quando o sistema opera em regime permanente, corrigindo a tendência existente no ONFC de crescimento dos pesos do controlador devido ao ruído. Este algoritmo é simples e pode ser implementado na grande maioria dos sistemas de controle industriais [Carvalho 2010], sendo proposta a minimização de uma nova função de custo, na qual o erro quadrático total $\left(J_{1}\right)$ passa a ser composto por duas parcelas: o erro de processo, proveniente das perturbações e mudanças de setpoint, e o efeito do ruído na ação de controle. O erro total é diretamente proporcional à diferença dos pesos $(D w)$ multiplicada pelo ruído do processo. Assim, busca-se minimizar o erro de processo, penalizando simultaneamente o crescimento excessivo dos pesos, conforme Equação 17.

$$
\min J_{1}=(1-\beta) \frac{1}{2} e^{2}+\beta \frac{1}{2}\left(w_{2}-w_{1}\right)^{2}
$$


$\mathrm{O}$ termo $\beta$ pode assumir qualquer valor entre 0 e 1 sendo utilizado para ponderar as parcelas de minimização do erro e regularização dos pesos. O procedimento para obtenção das equações de atualização dos pesos e a saída do controlador é o mesmo do ONFC original. Deriva-se $J_{1}$ em relação aos pesos:

$$
\frac{\partial J_{1}}{\partial w_{i}}=\frac{\partial J_{1}}{\partial e} \frac{\partial e}{\partial y} \frac{\partial y}{\partial u} \frac{\partial u}{\partial w_{i}}+\frac{\partial J_{1}}{\partial \Delta w} \frac{\partial \Delta w}{\partial w_{i}}
$$

A derivada da primeira parcela da Equação 18 é análoga ao obtido para o ONFC acrescentando apenas o termo multiplicativo $(1-\beta)$. Já a derivada da segunda parcela é dada por:

$$
\begin{gathered}
\frac{\partial J_{1}}{\partial \Delta w}=\beta\left(w_{2}-w_{1}\right) \\
\frac{\partial \Delta w}{\partial w_{1}}=\frac{\partial\left(w_{2}-w_{1}\right)}{\partial w_{1}}=-1 \\
\frac{\partial \Delta w}{\partial w_{2}}=\frac{\partial\left(w_{2}-w_{1}\right.}{\partial w_{2}}=1
\end{gathered}
$$

Assim, para o controlador $\mathrm{ONFC}_{D w}$, as equações de ajuste dos pesos são:

$$
\begin{aligned}
& w_{1}^{k}=w_{1}^{k-1}+(1-\beta) \alpha \mu_{1}^{k} e^{k}+\beta \alpha\left(w_{2}^{k-1}-w_{1}^{k-1}\right) \\
& w_{2}^{k}=w_{2}^{k-1}+(1-\beta) \alpha \mu_{2}^{k} e^{k}-\beta \alpha\left(w_{2}^{k-1}-w_{1}^{k-1}\right)
\end{aligned}
$$

Com a formulação do $\mathrm{ONFC}_{D w}$, o efeito de aumento da diferença dos pesos que poderia gerar a instabilidade no controlador é contornado. Sendo assim, essa versão do controlador utilizada no presente trabalho.

\section{Taxa de adaptação dinâmica}

O parâmetro $\alpha$, ou taxa de aprendizagem do ONFC, ajusta tanto o passo do método de otimização gradiente descendente, usado no ajuste dos pesos, quanto o desempenho do sistema em malha fechada.

A partir de simulações do comportamento de sistemas de primeira ordem para diferentes valores de $\alpha$, [Carvalho 2010] apresenta uma proposição para encontrar um alfa-ótimo. Utilizando Redes Neurais e ferramentas de otimização estocásticas, o autor apresenta uma metodologia de sintonia e projeto do ONFC quanto à taxa de aprendizado. Contudo essa metodologia utiliza um alfa fixo durante o controle do processo. Além disso, novos passos e parâmetros são criados na metodologia, aumentando a complexidade de projeto do controlador.

Uma estrutura do tipo NFN semelhante a do ONFC, porém aplicada a problemas de identificação, foi apresentada por [Caminhas et al. 1998]. Nesse trabalho o autor apresenta uma taxa de aprendizagem que permite que o método gradiente descente alcance 
um erro de aproximação nulo em apenas um passo para problemas de identificação de sistemas. O resultado foi empregado com sucesso para treinamento em tempo real. Nessa formulação, o $\alpha^{k}$ é calculado conforme equação 24 .

$$
\alpha^{k}=\frac{1}{\left(\mu_{1}^{k}\right)^{2}+\left(\mu_{2}^{k}\right)^{2}}
$$

Propõe-se então, aplicar a equação 24 utilizada em uma estrutura NFN para identificação em sistemas dinâmicos no $\mathrm{ONFC}_{D w}$, substituindo o numerador por um parâmetro $\alpha$, que varia de acordo com o modelo do processo a ser controlado, conforme equação 25 [Ferreira 2017].

$$
\alpha^{k}=\frac{\alpha}{\left(\mu_{1}^{k}\right)^{2}+\left(\mu_{2}^{k}\right)^{2}}
$$

O valor de $\alpha$ pode ser determinado empiricamente, com a ressalva que valores altos para $\alpha$ podem levar o sistema à instabilidade. No presente trabalho, não foi permitido que o valor de $\alpha$ ultrapassasse o valor 2, em analogia a outros trabalhos envolvendo controles adaptativos [Goodwin et al. 1980]. Empiricamente, o valor definido para $\alpha$ neste trabalho foi de 0,01 .

\section{Metodologia}

O controlador $\mathrm{ONFC}_{D w}$ com a nova abordagem da taxa de aprendizagem foi empregado no controle de vazão de um processo real. O processo onde o controlador foi utilizado é composto por dois tanques, no qual é realizado o controle da vazão de água que abastece o tanque superior, vinda do tanque inferior. No tanque inferior há uma bomba cuja velocidade é controlada com um inversor de frequência. A vazão de água que alimenta o tanque superior é medida. A água retorna do tanque superior para o tanque inferior por gravidade. Há também uma válvula de controle após a bomba, que será utilizada em alguns experimentos como perturbação para o controle.

Dois experimentos comparativos são propostos entre o controlador $\mathrm{ONFC}_{D w}$ com a taxa de aprendizagem dinâmica e um controlador PID sintonizado com a metodologia de Ziegler-Nichols. No primeiro, a válvula de controle fica toda aberta e são gerados vários setpoints de vazão, sendo que o controlador deve garantir que a vazão chegue o mais rápido possível ao setpoint, reduzindo ao máximo o overshoot de vazão. No segundo, o setpoint de vazão é mantido fixo e a válvula de controle é manipulada, gerando uma perturbação no sistema. Esse distúrbio deve ser rejeitado pelo controlador de tal forma que a vazão retorne ao setpoint o mais rápido possível.

\section{Resultados}

Os resultados para os experimentos de mudança de setpoint e para a rejeição de distúrbios são apresentados a seguir. No experimento de mudança de setpoints, a bomba é iniciada e após estabilização da vazão é gerado um conjunto de setpoints dentro da faixa de operação da planta. Os mesmos valores de setpoints foram utilizados com uso do controlador PID e também com o $\mathrm{ONFC}_{D w}$. A figura 3 apresenta os setpoints gerados em azul e a resposta 


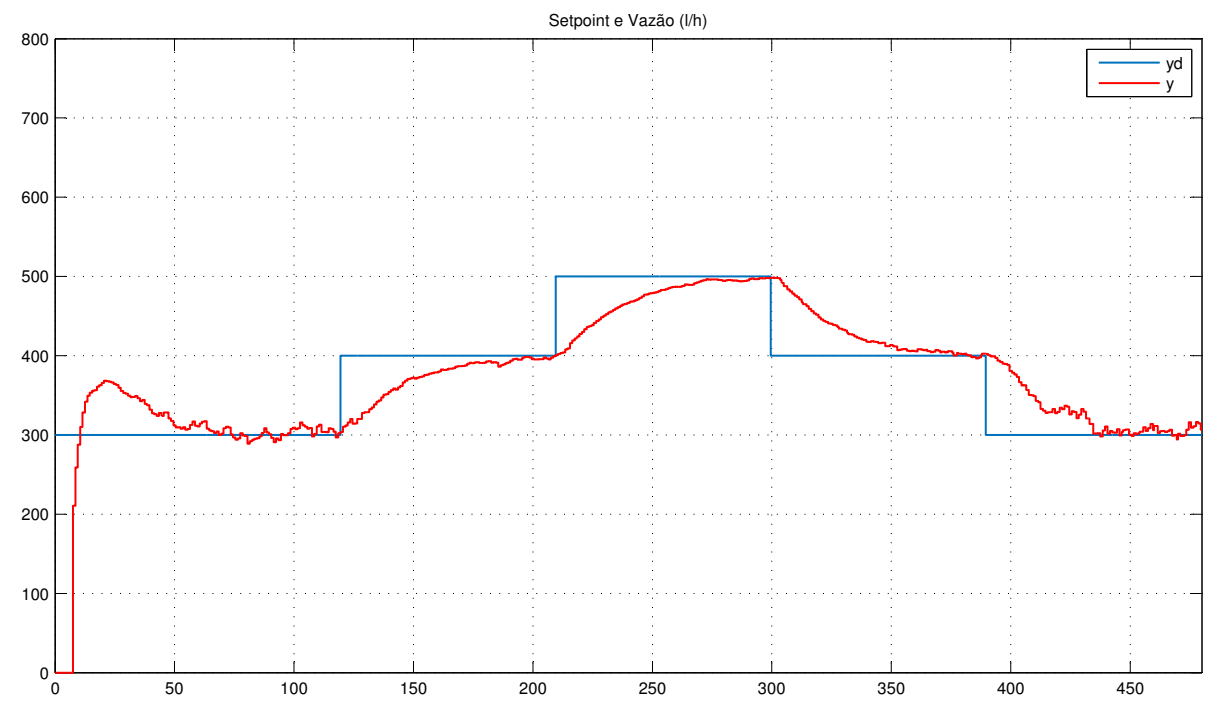

Figure 3. Resposta com Controlador PID.

da vazão com a utilização do controlador PID em vermelho. Neste experimento, a integral do erro absoluto obtida foi 29859.

A ação de controle, dada pelo percentual da velocidade do inversor de frequência, é apresentada na figura 4.

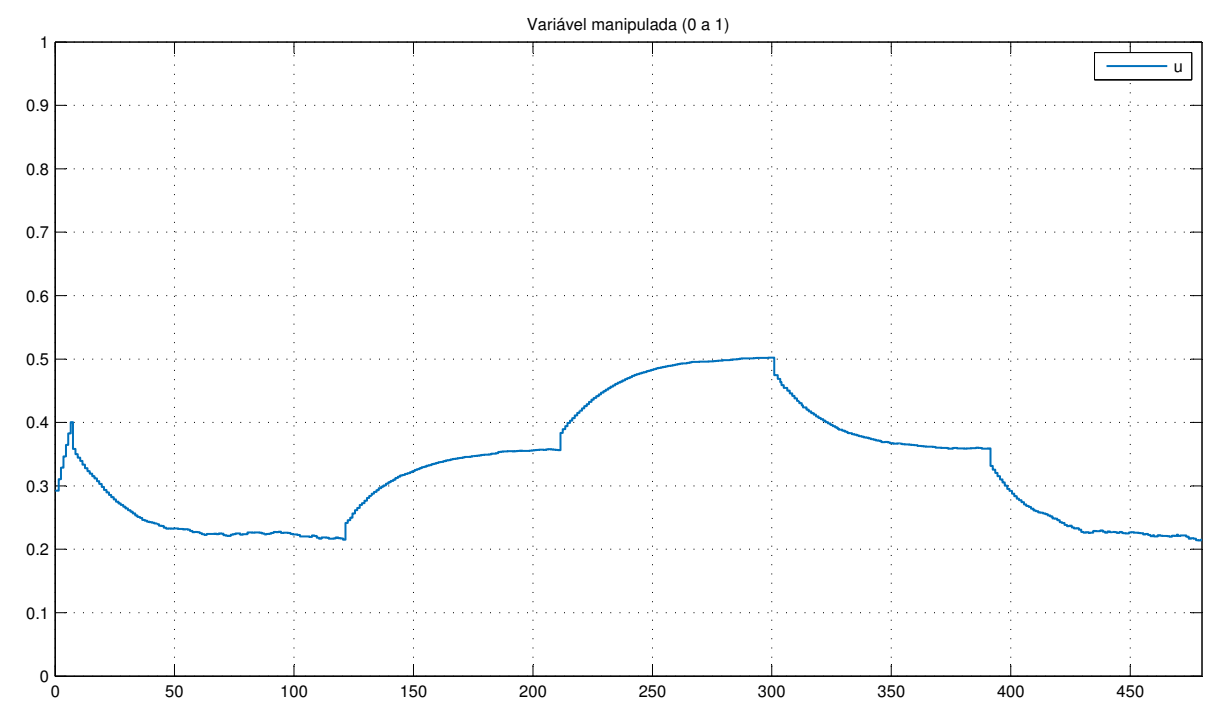

Figure 4. Saída do Controlador PID.

$\mathrm{O}$ mesmo experimento utilizando o controlador $\mathrm{ONFC}_{D w}$ gerou os resultados apresentados na figura 5, sendo os setpoints em azul e a vazão em vermelho. Neste experimento, a integral do erro absoluto obtida foi 16708 , aproximadamente $44 \%$ de redução em relação ao experimento com o controlador PID.

A ação de controle para o controlador $\mathrm{ONFC}_{D w}$ é apresentada na figura 6. 


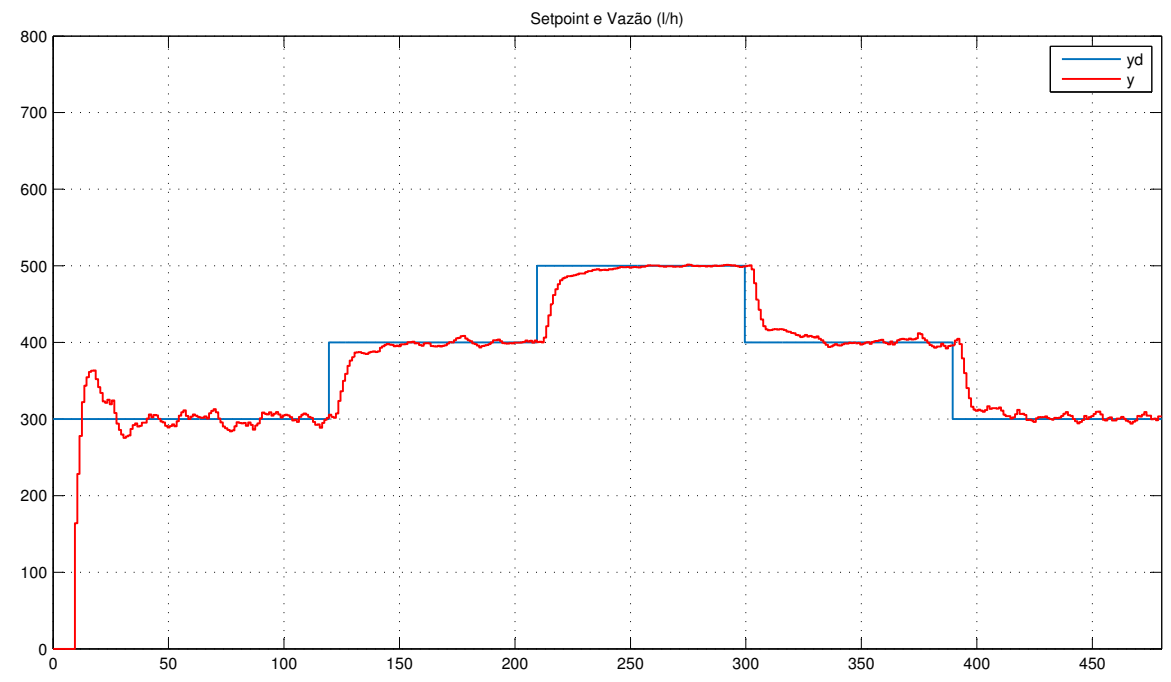

Figure 5. Resposta com ONFC ${ }_{D w}$ com nova abordagem para o parâmetro $\alpha$.

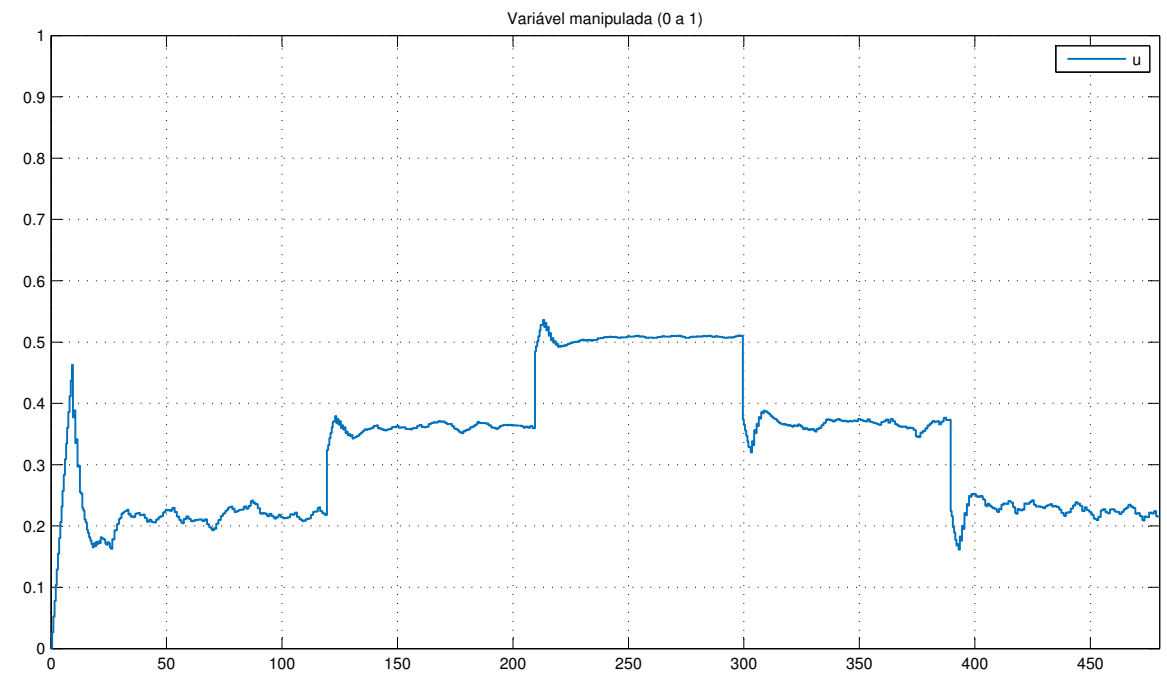

Figure 6. Saída do ONFC ${ }_{D w}$ com nova abordagem para o parâmetro $\alpha$.

Para o teste de rejeição de distúrbios, a válvula é mantida em $40 \%$ de abertura, sendo este o término da faixa linear da válvula e a vazão estabilizada pelo controlador em $500 \mathrm{l} / \mathrm{h}$. É gerado um distúrbio no processo a partir de uma redução brusca da válvula para $10 \%$ de abertura e é avaliado o comportamento do controlador para levar novamente a vazão até o setpoint de $500 \mathrm{l} / \mathrm{h}$. Na figura 7 é apresentado o comportamento da vazão após aplicar a perturbação com a ação do controlador PID. A integral do erro absoluto nesse experimento foi 10706. 


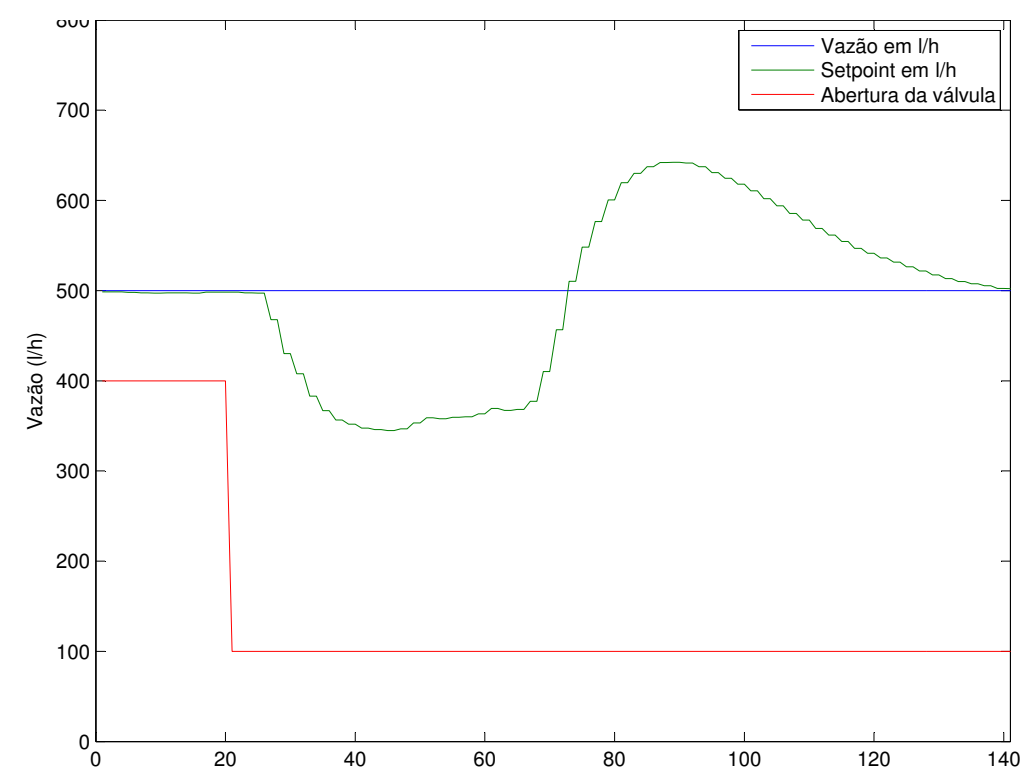

Figure 7. Controlador PID rejeitando distúrbio.

Na figura 8 é apresentada a vazão após aplicar a perturbação com a ação do controlador $\mathrm{ONFC}_{D w}$ com a taxa de aprendizagem dinâmica. A integral do erro absoluto nesse experimento foi 1391, uma redução de mais de $87 \%$ do valor do controlador PID.

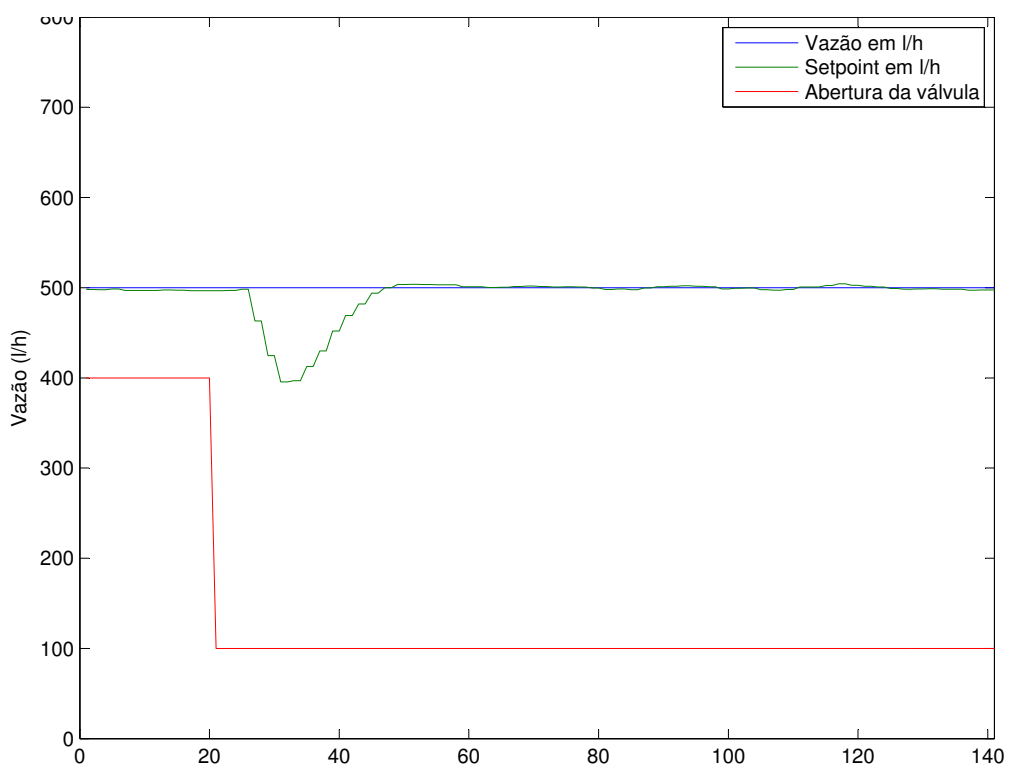

Figure 8. ONFC rejeitando distúrbio.

Apresentados os resultados do experimento, é possível verificar que o $\mathrm{ONFC}_{D w}$ com a taxa dinâmica adaptativa foi capaz de controlar a vazão do processo, tanto acompanhando as mudanças de setpoints quanto rejeitando distúrbios provocados pela redução da abertura da válvula de controle. Comparando com o controlador PID utilizado previamente na planta, sintonizado com o método de Ziegler-Nichols em malha aberta, nesse primeiro experimento observa-se um desempenho consideravelmente melhor, tanto para acompanhamento de mudanças de setpoints quanto para rejeição de distúrbios. 


\section{Considerações finais}

$\mathrm{O}$ presente artigo utilizou o controlador $\mathrm{ONFC}_{D w}$ para controle de vazão de água em processo real com a utilização de uma taxa de aprendizagem dinâmica. Para tanto, foi realizada uma revisão do controlador ONFC, que mostra-se um controlador de fácil implementação, possui poucos parâmetros e é adaptativo às condições do processo a ser controlado.

Para realização dos experimentos, foram criadas situações de mudança de setpoint da vazão do processo e também criação de distúrbios na planta, por meio da mudança na abertura de uma válvula de controle que interfere na vazão do processo. Em ambos os casos o controlador $\mathrm{ONFC}_{D w}$ com a taxa de aprendizagem dinâmica apresentou resultados de controle satisfatórios, ficando sempre estável durante os testes e com desempenho melhor que do controlador PID que era usado na processo, gerando menor overshoot, menor tempo de resposta e, consequentemente, menores valores para a integral do erro absoluto.

Contudo, foram realizados apenas experimentos iniciais para validação da taxa de aprendizado dinâmica, baseada em uma abordagem utilizada na literatura para sistemas NFN usados em identificação de sistemas dinâmicos. A utilização da taxa de aprendizagem dinâmica em outros experimentos poderá consolidar a metodologia para ajuste do parâmetro.

\section{Agradecimentos}

O presente trabalho foi realizado com o apoio financeiro da CAPES - Brasil.

\section{References}

Caminhas, W., Pereira, G., Tavares, H., and Gomide, F. (1998). Identificação de sistemas dinâmicos: Abordagem baseada em neurônio nebuloso. Anais do V Simpósio Brasileiro de Redes Neurais (SBRN 98), Belo Horizonte-MG, pages 105-110.

Carvalho, M. A. (2010). Controlador neurofuzzy com aprendizado on-line: Teoria e aplicação na indústria de petróleo. Master's thesis, Universidade Federal de Minas Gerais.

dos Santos, S. R., Amaral, J. L., and Amaral, J. F. M. (2016). Adaptive intelligent systems applied to two-wheeled robot. In Evolving and Adaptive Intelligent Systems (EAIS), 2016 IEEE Conference on, pages 146-150. IEEE.

Ferreira, C. Z. M. (2017). Controlador NeuroFuzzy Online: Estudo Comparativo com PID em Simulação e Aplicação Real. Monografia de Graduação. Universidade Federal de Minas Gerais.

Goodwin, G., Ramadge, P., and Caines, P. (1980). Discrete-time multivariable adaptive control. IEEE Transactions on Automatic Control, 25(3):449-456.

Gouvêa, M. R. (2005). Controle Neurofuzzy de Motor de Indução com Estimação de Parâmetros e Fluxo de Estator. PhD thesis, Universidade Federal de Minas Gerais.

Jang, J.-S. R., Sun, C.-T., and Mizutani, E. (1997). Neuro-Fuzzy and Soft Computing. Prentice Hall. 
Ogata, K. (2003). Engenharia de Controle Moderno. Pearson Prentice Hall.

Pires, A. V. (2007). Controladores baseados em técnicas de inteligência computacional: Análise, projeto e aplicações. Master's thesis, Universidade Federal de Minas Gerais.

Precup, R.-E. and Hellendoorn, H. (2011). A survey on industrial applications of fuzzy control. Computers in Industry, 62(3):213-226.

Yamakawa, T., Uchino, E., and Miki, T. (1992). A neo fuzzy neuron and its application to system identification and prediction of the system behaviour. Proceedings of the Second International Conference on Fuzzy Logic and Neural Networks, (2):477-483. 